\title{
Surface Registration for Use in Interactive Image- Guided Liver Surgery
}

\author{
Alan J. Herline ${ }^{1}$, Jeannette L. Herring ${ }^{2}$, James D. Stefansic ${ }^{3}$, William C. Chapman ${ }^{1}$, \\ Robert L. Galloway, and Benoit M. Dawant ${ }^{2}$ \\ ${ }^{1}$ Division of Hepatobiliary Surgery and Liver Transplantation \\ ${ }^{2}$ Department of Electrical and Computer Engineering \\ ${ }^{3}$ Department of Biomedical Engineering \\ Vanderbilt University, 801 Oxford House, Nashville, TN 37235 \\ alan.j.herline@vanderbilt.edu
}

\begin{abstract}
Liver surgery is difficult because of limited external landmarks, significant vascularity and inexact definition of intrahepatic anatomy. Intraoperative ultrasound (IOUS) has been widely used in an attempt to overcome these difficulties, but is limited by its two-dimensional nature, interuser variability and image obliteration with ablative or resectional techniques. Because the anatomy of the liver and intra-operative removal of hepatic ligaments make intrinsic or extrinsic point-based registration impractical, we have implemented a surface registration technique to localize physical points for liver phantoms and anatomically placed targets within the liver on CT images. Liver phantoms were created from anatomically correct molds with "tumors" imbedded within the substance of the liver. Helical CT scans were performed with $3 \mathrm{~mm}$ slices. Using an optically active position sensor, the surface of the liver was digitized according to anatomical segments. A surface registration was performed and RMS errors of the locations of internal tumors are presented as verification. An initial point based marker registration was performed and considered as the standard for error measurement. Errors for surface-registration were $2.9 \mathrm{~mm}$ for the entire surface and $2.8 \mathrm{~mm}$ for embedded targets. This is an initial study considering the use of surface registration for the purpose of physical to image registration in the area of liver surgery.
\end{abstract}

\section{Introduction}

Liver surgeons have relied on preoperative imaging studies to select patients for operative intervention. The "operative planning", however, has been performed in an inexact and general conceptual fashion (i.e., removing the right lobe versus a wedge resection of the right lobe) as opposed to a more precise resection. A recent lead article in the Annals of Surgery suggested that preoperative planning with 3dimensional CT reconstructions and virtual-reality techniques could allow precise preoperative planning and even allow surgeons to "practice" the resection and perhaps minimize operative errors in the actual resection procedure $[1,2]$. While this approach could provide CT visualization of planned liver resections, no one has reported a method to execute such an image-guided pre-planned hepatic resection. 
One suggested method to provide tomographic information for surgical guidance involves operating room placement of scanners [3]. However, these systems have significant barriers to broad acceptance: 1) These units are very costly on the basis of installation, per procedure costs and intra-operative modification of instruments to avoid magnetic interference. 2) These systems are large and can make standard operations more difficult because of space occupied by imaging equipment. We have initiated studies investigating alternative methods of intra-operative guidance for liver surgery that we believe will provide more tomographic data and be much more cost effective than placing scanners in the OR.

There are a number of initial engineering problems in adapting current guidance systems from neurosurgical procedures for use in hepatic procedures. Given these constraints, obtaining the margin of error demonstrated in neurosurgical systems is probably not feasible and is not needed for hepatic applications. The current gold standard for intra-operative localization is intra-operative ultrasound (IOUS). IOUS has the ability to accurately identify tumors of $5 \mathrm{~mm}$ or greater [2]. The liver has a variety of ligamentous attachments to the diaphragm and therefore physical space position is also dependent on the point in the respiratory cycle. The liver deforms and its thin capsule does not add rigidity. Point based registration methods are difficult to implement secondary to the inability to attach markers to the liver clinically. Even intrinsic point based methods become impractical due to the lack of points visible on the pre-operative tomographic images that would then be visible to the surgeon intraoperatively. Initial attempts at this method led to registration errors in the $12 \mathrm{~mm}$ range. Given our experience in the area of registration and more recently in the area of surface-based registration, we sought to determine the feasibility of surface registration as a method for physical to image registration for the liver $[4,5,6]$.

\section{Methods}

\subsection{Phantom, Target Creation and Image Acquisition}

Phantom livers were constructed from a two component poly (dimethyl) siloxane (rubber silicone). The livers are poured into a plaster mold allowing for the addition of "tumors". The current phantom tumors are cork of various sizes from $4-15 \mathrm{~mm}$. In addition to tumors we have placed 4 markers in the liver phantom so that they are evenly spaced and located in various anatomical segments. The marker system consists of plastic marker posts that are attached to the phantom, imaging markers that generate a high intensity level in CT images, and physical-space markers that contain a hemispherical divot into which the ballpoint tip of a three-dimensional spatial localizer (3DSL) fits precisely [7]. The position of an image marker is defined as its centroid and is determined using the algorithm described in [7]. The liver is scanned using a helical CT scanner, Philips Tomoscan AV scanner with 3mm slices and voxel dimensions of $0.50774 \mathrm{~mm} /$ pixel. For these experiments the 6 "tumors" are easily visualized on the images. 


\subsection{Physical Surface and Point Collection}

The physical surface of the liver is digitized using an optically active position sensor and a 3DSL. An optically active position sensor (Optotrak 3020, Northern Digital, Ontario, Canada) is used to detect the IREDs and it can precisely calculate the position of the tip if four of the IREDs are visible. This system is used to locate the position of the markers in physical-space, which correspond to the centroid of the image markers in the CT images. Approximately 900 points are collected from the entire anterior surface of the liver using a probe with a $3 \mathrm{~mm}$ tip in a manner that could be duplicated in the operating room. Following this and prior to any movement of the liver the surfaces of each of the visible 8 anatomic segments of the liver (figure 1) are collected in consecutive trials of $10-15$ seconds and $200-300$ points per trial. The final set of data is collected from the edges of each of the 8 visible segments. The image markers placed on the liver phantom are then removed and replaced with physical

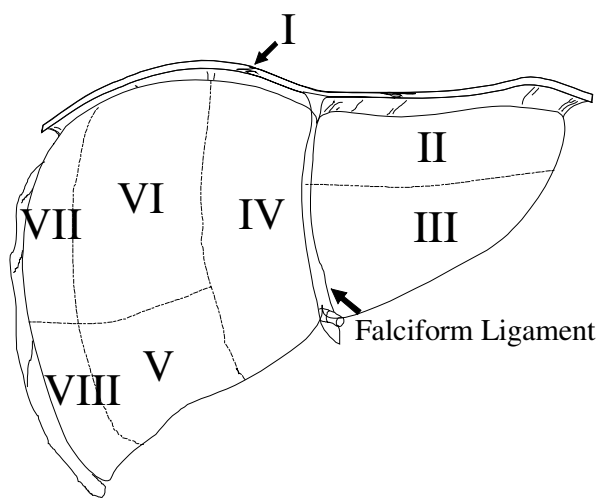

Fig. 1. Liver with the anatomical segments drawn upon the anterior surface. These segments correspond to blood supply for the liver. Segment I is not generally visible and is located on the liver's undersurface.

markers and these points are collected. These points are used for initialization of the surface based registration. The "gold standard" registration is performed using the points as described in [8].

\subsection{Image Segmentation and Surface Creation}

The phantom liver and the "tumors" are semi-automatically segmented using Midas (in-house image processing software). A triangle set representation of the liver surface is automatically generated using an independently implemented version of the classic marching cubes method developed by Lorensen and Cline [9], and the final representation was generated by simplifying the surface produced by the marching cubes algorithm using the VTK vertex-deletion scheme [10]. The resulting triangle set represents a close approximation to an iso-surface that is characterized by a specific intensity value. This iso-intensity-based approach is well suited to the extraction of the surface of a phantom imaged in isolation, since there are no 
surrounding tissues to confuse the definition of the surface. The only parameter that the triangulation algorithm requires is an intensity value. The Hounsfield number of the phantom is approximately 400, and the Hounsfield number of the air surrounding the phantom is -1024. There is an offset of 1024 introduced into the intensity values due to the method used to transfer the scans from the CT machine, resulting in average intensity values of about 1400 for the phantom and 0 for the surrounding air. We chose to use an intermediate value of 600 as an input to our intensity-based surface extraction algorithm. A different segmentation method will be required for use with a patient liver.

\subsection{Registration}

We initially perform a point-based registration using the method described by Maurer [8]. This serves as a "gold standard" for comparison of the surface-based registrations to follow. We perform surface-based registration using an independent implementation [11] of the iterative closest point registration algorithm of Besl and McKay [12]. First, the closest point on one surface is computed for each point in a set of points representing the other surface. In this study, the first surface is a triangle set representation of the liver surface in the $\mathrm{CT}$ image, and the point set representation of the second surface is a set of physical space surface points. Second, a transformation is determined by registering these two point sets. This process is iterated until some stopping criterion is satisfied. This method converges to a local minimum of the cost function, which is the root-mean-square (RMS) distance between the corresponding points at the last iteration. Because the physical space surface points we record are the positions of the center of the ballpoint tip of the 3DSL, the recorded surface points are displaced from the actual surface by the radius of the tip. We have not corrected for this and it is a source of error.

Because of the possibility of convergence to a local minimum that is not the correct solution, the algorithm works best when it is initialized with rotations and translations that are close to the exact solution. In this work, the initial registration for the physical points is computed by using the point-based algorithm with the markers and then rotating and translating those points from a corner of the image by progressive amounts for each trial to test the sensitivity of the algorithm to initial conditions.

\subsection{Error Calculation}

To evaluate the registration error obtained with the surface-based registration method we propose, the following procedure was utilized. First, the four fiducial markers were used to compute a rigid-body transformation between physical and image space using point-based registration of the marker centroids, which were located as described in section 2.2. The image-space coordinates of all the physical points acquired with the 3DSL were then computed using this transformation. For each registration trial performed with the surface-based method being investigated, a physical-to-image transformation matrix was generated and used to compute a second set of image-space coordinates for the physical points. The registration error obtained with the surface-based approach was then computed as the root-mean-square (RMS) 
difference between the image coordinates obtained with the marker-based registration and the coordinates obtained using our surface-based approach.

A more clinically applicable error is calculated using the position of targets more central within the volume of interest instead of surface points. Our results are examined by looking at the RMS error for both the surface and each of six tumors embedded within the liver phantom for the following cases: 1. surface registration using the total surface, 2. using an individual liver segment's surface 3. using an individual liver segment's surface boundary or edge.

For RMS calculations of target registration errors (TREs) for the six tumors, the CT image points corresponding to each tumor are transformed into physical space using two different transformations. Each tumor is transformed from image space into physical space using the results of the point-based registration. These points establish a "gold standard" position for the tumor. Then, each tumor is transformed from image space into physical space using the results of the surface-based registration which is being tested in these experiments. RMS error is then calculated from these two point sets, and this RMS error is designated the TRE for the tumor.

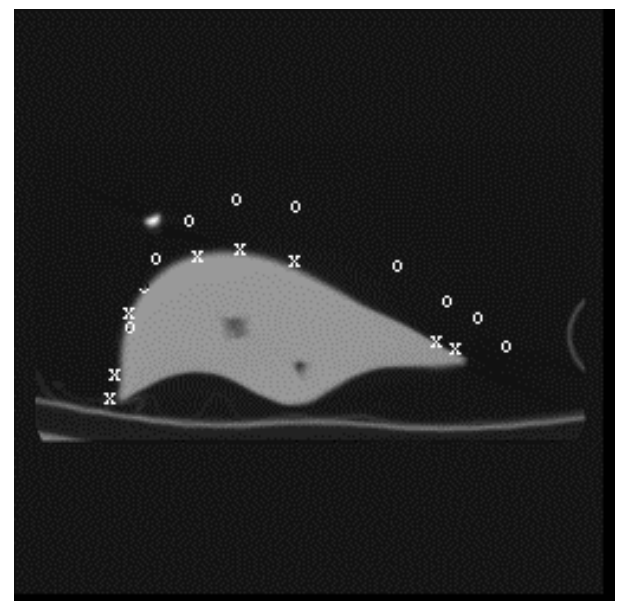

Figure 2. Shown is a CT scan of the phantom liver with o's showing the starting point for the 15 degree rotation and x's showing the results of the surface registration.

\section{Results}

\subsection{Surface Error}

Using the markers and posts embedded onto the substance of the liver we obtained a fiducial registration error (FRE) of $0.95 \mathrm{~mm}$. Individual registrations were performed using nine surfaces (eight segments and a total surface) and eight segment boundaries (or edges) for the following three trials: 1. Rotation of 5 degrees and translation of 5 $\mathrm{mm} 2$. Rotation of 10 degrees and translation of $5 \mathrm{~mm} \mathrm{3}$. Rotation of 15 degrees and 


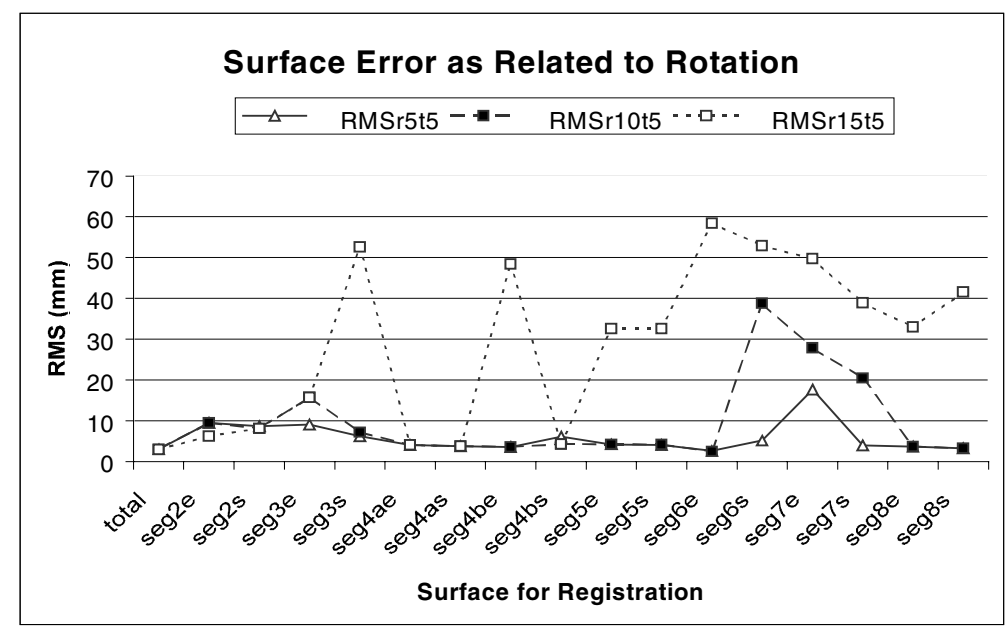

Fig. 2. RMS error for the entire surface using the total surface and various surface segments and their boundaries for registration.

translation of $5 \mathrm{~mm}$. A total of 51 trials were performed to study the RMS error of surface registration with the above points and under the listed starting locations of the physical point set. RMS error is calculated for the entire surface. This is demonstrated on a single slice of the CT scan in figure 1.

The graph for all results is shown in figure 2. The average RMS error using the total surface for registration was $2.99 \pm 0.006 \mathrm{~mm}$ for the three trials. Using segment VI boundary produced smaller RMS errors $(2.6,2.5 \mathrm{~mm})$ for the trials with 5 degrees and 10 degrees of rotation, respectively. The 15 degree rotation trial produced a 58.4 mm RMS for the segment VI boundary.

\subsection{Target Error}

Individual registrations were again performed using the nine surfaces and eight segment boundaries as above for the same three rotations and translations of initial points. RMS errors were calculated on six tumors distributed within the phantom in a fashion similar to clinical tumors. RMS errors were calculated for 306 trials differing in tumor localized, surface used for registration and rotation of initial points. The graph for all results is shown in figure 3. The average RMS error for all six tumors using the total surface for registration with all three rotations as initial points was $2.82 \mathrm{~mm} \pm 0.3 \mathrm{~mm}$. The range in error was from $2.45-3.35 \mathrm{~mm}$. 


\section{Discussion}

Surface registration has been implemented and used for neurosurgical applications because of the potential for accurate registration in a non-invasive, retrospective manner. While effective from a retrospective standpoint, a good surface fit may not offer a similar level of accuracy for objects beneath the surface. While extrinsic point-based techniques offer robustness appropriate for neurosurgical applications, they are difficult to implement for organs not surrounded by osseous formations and errors are difficult to quantify for intrinsic point-based methods [8]. Our interest has been to develop an image-guided system for hepatic resection and ablation of liver tumors. The liver is a smooth organ with few surface markings. It is not amenable to applied markers or suitable for accurate intrinsic point-based registration. The anatomical location of hepatic tumors is usually determined pre-operatively by tomographic imaging (CT or $\mathrm{MR}$ ). Ultrasonography can provide intraoperative imaging information regarding tumor location and surrounding vascular anatomy [2], but it requires special expertise to perform and properly interpret. We are investigating the use of endoscopic registration, but feel that surface registration may offer an effective method given the physical constraints of our clinical situation [4].

The errors obtained for tumor localization in the current study are very acceptable for hepatobiliary applications. The small variance between different trials when using a total surface for registration indicates the insensitivity of the algorithm to initial conditions. For transformations calculated only using a segment of the liver's surface we observed the following: 1) as rotations increased error increased dramatically. 2) For those segments with smaller error there was a wider range of error between tumors. Both of these suggest that a single segment's surface may not be adequate for the entire volume of the liver; however, it may not be necessary to obtain a surface from the entire liver to be accurate for a tumor isolated to a single segment.

Our errors can be attributed to several known problems. The centroid of the probe tip is $1.5 \mathrm{~mm}$ from the surface of the liver. This can be corrected, but was not in this study. While this correction would increase the accuracy for this study, it may not approach clinical reality as the surface of the liver is deformable even under slight pressure and a consistent surface to 3DSL point distance may not be known. Additionally the presumed physical space locations of the tumors are obtained by the point-based registration using the markers. This is a problem of having centrally located targets whose exact physical locations are not known and error is introduced using this method. We feel tracked ultrasound will be a feasible method to verify the registrations for deeper structures and our group has begun preliminary investigations in this area [13].

\section{Conclusions}

We are encouraged by our initial work in the use of surfaces from a liver phantom. This work can potentially apply the use of image-guidance for hepatic procedures. There is further design and experiments needed to clarify the amount of surface needed in order to adequately register the liver volumes with physical space and in the area of target design and localization for this application. 


\section{Acknowledgements}

The authors want to acknowledge support from the NSF, BES-9703714 and the NIH NRSA \#1 F32 DK 09671-02 SB. They want to thank Cindy Duncan, CRT and Tina Herron, CRT for their invaluable assistance with imaging.

\section{References}

1. Marescaux, J., et al., Surgical simulation and virtual reality: the coming revolution. Ann Surg, 1998. 5: p. 635-637.

2. Rafaelsen, S.R., et al., Intraoperative ultrasonography in the detection of hepatic metastases from colorectal cancer. Dis Colon Rectum, 1995. 38: p. 355-360.

3. Klotz, H., R. Flury, and P. Erhart, Magnetic resonance-guided laparoscopic interstitial laser therapy of the liver. AJS, 1997. 174: p. 448-451.

4. Herline, A., et al., Studies in the Feasibility of Interactive Image-Guided Liver Surgery. Arch. Surg., 1999.

5. Herring, J.L., et al., Surface-based Registration of CT Images to Physical Space for Image-guided Surgery of the Spine: A Sensitivity Study. IEEE Trans. Med. Imag., 1998. 17: p. 743-752.

6. Herring, J.L., et al. Effect of vertebral surface extraction on registration accuracy: a comparison of registration results for iso-intensity algorithms applied to computed tomography images. in SPIE. 1999. San Diego, CA.

7. Wang, M.Y., et al., An automatic technique for finding and localizing externally attached markers in CT and MR volume images of the head. IEEE Trans. Biomed. Eng., 1996. 43: p. 627-637.

8. Maurer, C.R., et al., Registration of head volumes using implantable fiducial markers. IEEE Trans. Med. Imag., 1997. 16: p. 447-462.

9. Lorensen, W.E. and H.E. Cline, Marching Cubes: A High Resolution 3D Surface Construction Algorithm. Computer Graphics, 1987. 21: p. 163-169.

10. Schroeder, W., M. K., and L. V., The Visualization Toolkit. 1998, Upper Saddle River, New Jersey: Prentice Hall PTR.

11. Maurer, J.C.R., et al., A method for registration of 3-D images using multiple geometrical features. IEEE Trans. Med. Imag, 1996. 15: p. 836-849.

12. Besl, P.J. and N.D. McKay, A method for registration of 3-D shapes. IEEE Trans. Pattern Anal. and Machine Intell, 1992. 14: p. 239-256.

13. Beasley R. A., S.J.D., Herline A.J., Guttierez L., Galloway Jr., R. L.. Registration of ultrasound images. in SPIE. 1999. San Diego, CA. 